\title{
Nutzenbewertung der Off-Label-Praxis in Zusammenarbeit mit Kompetenzzentren für klinische Forschung
}

\author{
Sicherheit und Wirksamkeit von Off-Label-Medikamenten müssen sorgfältig eruiert \\ werden. Clinical Trial Units könnten dabei einen wesentlichen Beitrag leisten und \\ zukünftig ihre studientechnischen Kenntnisse und ihre Infrastruktur der Off-Label- \\ Forschung zur Verfügung stellen.
}

Titus Bihl

Leitender Arzt Rheumatologie, Physikalische Medizin und Rehabilitation HFR Kantonsspital Fribourg
Dieser Artikel ist Teil der Abschlussarbeit des Postgraduate Educational Programms

«University Professional in Clinical Trial Practice and Management» Kurs 2010-2012 der Universität Basel realisiert duch die Clinical Trial Unit (CTU) Basel.

Herzlich danken möchte ich Frau Dr. Barbara Christiane Peters für die Organisation des hervorragenden Kurses. Ein Dank geht auch an Frau Prof. Christiane Pauli-Magnus, Direktorin der CTU Basel, für die Anregung und Durchsicht dieser Arbeit.

Korrespondenz: Dr. med. Titus Bihl HFR Kantonsspital CH-1700 Fribourg Tel. 0264267385 Fax 0264267387 bihlt[at]h-fr.ch

\section{Ausgangslage}

Seit Fichte (1792-1814) wissen wir, dass unsere Begriffsbildung mit dem Prinzip des dialektischen Widerspruchs verwoben ist [1]. Weiss man, was z. B. unter den Begriff Label fällt, dann weiss man auch, was nicht darunter fällt.

Das Label ist heute gut definiert und entspricht der Fachinformation, die gleichsam die Summe gültiger, wahrer und unvoreingenommener Datensätze ist und für pharmazeutische Qualität, medizinische Evidenz, ausreichend Information über Nebenwirkungen, bekanntes Risiko-Nutzen-Verhältnis, gut lesbare Benutzerinformation, Verbraucherschutz und vieles mehr steht. Alles andere ist zu Beginn der 80er Jahre unter den Begriff Unlabeled use zusammengefasst worden. Erst 1988 taucht schliesslich der heute übliche Begriff Off-Label auf [2,3], der seither für die Negation dessen, was in der Fachinformation zusammengefasst wird, steht. Und daher ist es nicht verwunderlich, dass sowohl von ethischer, politischer, juristischer und ökonomischer Seite nach einer näheren Bestimmung gefragt oder nach Modellen gesucht wird, die mehr Klarheit in die KostenNutzen- [4] oder die Risiko-Nutzen-Bewertung der Off-Label-Medikamente bringen.

Das Hauptproblem liegt darin, Information über den Nutzen eines Off-Label-Medikaments mit der Kontrolle seiner Verschreibung zu verknüpfen, ohne die Therapiefreiheit des Arztes einzuschränken [5].

\section{Wissenschaftliche Dimension}

Wie aus dem Obigen hervorgeht, bekommt man bei der Off-Label-Praxis das Gefühl, mit etwas «Unsicherem» konfrontiert zu sein. Man findet sich in einer Art «Spielraum des Unbestimmten», der die Bedingung der Möglichkeit für wissenschaftliche Forschung liefern könnte [6]. Mit jedem Einsatz scheint man neue Erkenntnisse zu generieren. Dies betonen auch die beiden Juristen Sprecher und Spyk, die aus juristischer Sicht den potentiellen Wissensgewinn mit dem Wissensmangel (Schadenspotential), der im Moment der Verschreibung vorliegt, verbinden

\section{Evaluation scientifique de la} pratique hors étiquette adaptée au risque en collaboration avec des centres de compétence de recherche clinique

Du point de vue juridique, économique, mais aussi déontologique, la pratique hors étiquette devrait être réglementée sans restreindre la liberté thérapeutique du médecin. C'est en attribuant à cette pratique une dimension scientifique que la prescription hors-étiquette se transforme en un essai à effectif unique qui contribue à combler ce qu'on appelle l'écart entre ce que nous savons et ce que nous faisons. Afin de rendre cette nouvelle pratique acceptable, il faut toutefois une stratification selon la présence ou l'absence de certains facteurs. C'est uniquement pour les prescriptions à haut risque qu'une démarche scientifique sera nécessaire pour définir le risque-bénéfice ou le coût-bénéfice. Les centres de recherche clinique (CRC) joueront le rôle d'interlocuteur pour toutes les questions tournant autour de cette recherche clinique. En même temps ils surveilleront cette pratique sans limiter la liberté thérapeutique.

[7]. Sie grenzen drei mögliche Therapiesituationen ab: die sichere Standardbehandlung, den Heilversuch im Rahmen der ärztlichen Freiheit und die gefährlichen wissenserweiternden experimentellen Einzelfallbehandlungen.

Während jedoch der einfache Heilversuch anfällig ist auf selbsterfüllende Prophezeiungen sei- 
Tabelle 1

Risiko- und Nutzenbeurteilung aufgrund von Studiendaten.

\begin{tabular}{lll} 
Quelle & Sicherheit & Wirksamkeit \\
\hline Phase-III-(IV-)Studien & Hohe & Hohe \\
\hline Phase I-II-Studien & Mässige & Mässige \\
\hline Präklinische Daten & Geringe & Geringe \\
\hline Keine Daten oder & Keine & Unklare \\
Konträre Evidenz & &
\end{tabular}

Konträre Evidenz

tens des Arztes (Rosenthal-Effekt) oder seitens des Patienten aufgrund einer sozial asymmetrischen Beziehung (Hawthorne-Effekt) oder einer gesellschaftlichen Erwartung (Andorra-Effekt), kann das Einzelfallexperiment ein systematisches, methodengeleitetes Vorgehen gewährleisten und somit der wissenschaftlichen Überprüfung einer Hypothese dienen.

Die Geriater Price und Evans führen näher aus, wie der individuelle Nutzen mittels wissenschaftlichem Einzelfallexperimenten festzustellen ist [8]. Als Kriterien, ein solches Experiment durchzuführen, listen sie den begründeten Zweifel an der Wirksamkeit, ein ungünstiges Nutzen-Risiko-Verhältnis basierend auf Ergebnissen aus Gruppenstudien und Langzeittherapien auf, deren Effizienz über die Zeit nachlassen können. Sie integrieren aber auch pharmako-ökonomische Überlegungen. Einzelfallexperimente definieren sie als planmässige und replizierbare Untersuchung eines Patienten, bei dem mindestens zwei Therapien (unabhängige Variable, z. B. Off-Label-Medikament, Plazebo oder zugelassene Therapie) nach dem Zufallsprinzip wiederholt (Randomisierung) und ohne Wissen des Therapeuten oder Patienten manipuliert werden (Blinding).

Um den Forschungscharakter zur Gewohnheit des praktizierenden Arztes zu machen und um als kontrolliertes Einzelfallexperiment von der Gemeinschaft der Wissenschaftler akzeptiert zu werden, muss sich diese zeit- und ressourcenintensive Methodologie der Häufigkeit und Variabilität der Off-LabelPraxis anpassen.

Tabelle 2

Modulatoren der Sicherheitsbeurteilung.

\begin{tabular}{lll} 
Indikatoren & Beschreibung & Einfluss \\
\hline Population & $\begin{array}{l}\text { Vulnerable Patienten- } \\
\text { gruppe }\end{array}$ & Verringert \\
\hline Dosis/Applikationsform & $\begin{array}{l}\text { Suboptimal } \\
\text { Überdosis }\end{array}$ & Erhöht \\
& Lokal statt systemisch & Erhöht \\
\hline Systemisch statt lokal & Verringert \\
& Längere Intervalle & Erhöht \\
\hline Kürzere Intervalle & Verringert \\
\hline Qualifikation & Spezialist & Erhöht \\
\hline & Nicht-Spezialist & Verringert
\end{tabular}

\section{Risikoanalyse}

In Anlehnung an das Modell von Brosteanu et al. [9] soll in einem ersten Schritt die Basissicherheit und Basiswirksamkeit des Off-Label-Medikaments qualitativ bestimmt werden. Dazu werden wissenschaftliche Studien, die zur Zulassung des Medikaments geführt haben, herangezogen. In präklinischen Studien werden die Moleküle in Labortests und an Tieren auf Wirkung und Sicherheit geprüft. Phase-IStudien untersuchen an einer geringen Anzahl Gesunder oder Patienten die ordnungsgemässe Dosis bzw. Menge des Arzneimittels sowie vorherrschende Nebenwirkungen. In der Phase II erhebt man Daten zur Sicherheit und zum Nutzen einer Behandlung an Patienten, die an der Zielkrankheit leiden. Phase-IIIStudien testen ebenfalls die Wirksamkeit und Nebenwirkungen, allerdings an einer ausreichend hohen Anzahl an Patienten, so dass auch seltenere Nebenwirkungen entdeckt werden können. Oftmals wird das neue Produkt auch mit einer bestehenden Behandlung verglichen, um herauszufinden, welche Therapie besser ist. Phase-IV-Studien liegen definitionsgemäss beim Off-Label-Medikament noch nicht vor (Tabelle 1).

\section{Modulatoren der Sicherheit}

In einem zweiten Schritt werden Indikatoren gesucht, die einen modulierenden Effekt auf die Grundsicherheit haben können. Gehört der Patient z. B. einer vulnerablen Patientenpopulation an, dann muss die Sicherheit nachhaltig in Frage gestellt werden. Unter vulnerablen Patienten versteht man Menschen, deren Urteils- und Kritikfähigkeit irgendwie kompromittiert ist, so dass sie einen besonderen Schutz brauchen. Die für das Off-Label-Medikament gewählte Dosis oder Applikationsform kann auf die Sicherheit genauso einen Einfluss haben wie die Qualifikation des Arztes (Tabelle 2).

\section{Modulatoren der Wirksamkeit}

In einem dritten Schritt werden Modulatoren gesucht, die die Basis-Wirksamkeit beeinflussen können. Beurteilt werden muss, ob das Off-Label-Medikament nur für ein anderes Stadium der In-labelTherapie erfolgt, für eine Subgruppe von Patienten oder für eine ganz andere Krankheit. Ferner kann das Geschlecht eine Rolle spielen (Gendermedizin), v.a. wenn es kardiologische, antiepileptische oder Mittel zur Blutverdünnung betrifft. Auch die Dosis und die Applikationsform können einen nicht zu unterschätzenden Einfluss auf die Wirksamkeit, aber auch auf die Sicherheit haben. Je mehr sie von der Standarddosis oder -applikationsform abweicht, desto zweifelhafter und gefährlicher wird das Off-LabelMedikament (Tabelle 3).

\section{Zusammenfassung der Risikoanalyse}

Die in der Risikoanalyse gewonnenen Informationen werden zusammenfasst, indem das Off-Label- 
Tabelle 3

Modulatoren der Wirksamkeitsbeurteilung.

\begin{tabular}{|c|c|c|}
\hline Indikatoren & Beschreibung & Einfluss \\
\hline \multirow[t]{3}{*}{ Indikation } & $\begin{array}{l}\text { Stadium (Früh-, Spät-, } \\
\text { fortgeschrittenes }\end{array}$ & Erhöht/verringert \\
\hline & $\begin{array}{l}\text { Entität (Subgruppe } \\
\text { der Erkrankung) }\end{array}$ & Erhöht/verringert \\
\hline & $\begin{array}{l}\text { Andere Erkrankung } \\
\text { (ähnlich - unähnlich) }\end{array}$ & Verringert \\
\hline Geschlecht & $\begin{array}{l}\text { Anderes Geschlecht als } \\
\text { <in-label>-Einsatz }\end{array}$ & Erhöht/verringert \\
\hline Dosis/ & Systemisch statt lokal & Verringert \\
\hline \multirow[t]{6}{*}{ Applikationsform } & Suboptimal & Verringert \\
\hline & Überdosis & Erhöht \\
\hline & Lokal statt systemisch & Verringert \\
\hline & Systemisch statt lokal & Erhöht \\
\hline & Längere Intervalle & Verringert \\
\hline & Kürzere Intervalle & Erhöht \\
\hline Generikum & Ökonomische Variante & Erhöht/verringert \\
\hline
\end{tabular}

Medikament einer der folgenden vier Risikogruppen zugewiesen wird:

A Sicher und hochwahrscheinlich wirksam

B Sicher, aber Wirksamkeit unklar

C Sicherheit, aber hochwahrscheinlich wirksam

D Unsicher und möglicherweise unwirksam.

Fällt das Off-Label-Medikament in die Risikoklasse A, dann braucht es kein wissenschaftliches Einzelfallexperiment, sondern es handelt sich um eine Arzneimittel-Therapie, die aus irgendwelchen Gründen keine offizielle Marktzulassung für diese Indikation hat.

\section{Die Off-Label-Praxis ist nicht durch Gesetze}

\section{oder staatliche Zulassungsbehörden zu kontrollieren.}

In der Risikoklasse B liegen genügend positive Modulatoren für die Sicherheit vor (s. Tabelle 2), aber wenig Quellen, die die Wirksamkeit dokumentieren. In einem solchen Fall soll die Off-Label-Praxis in einem kontrollierten Einzelfallexperiment überprüft werden. Dadurch gelingt es, den höchsten individuellen Nutzen zu bestimmen.

Fällt das Off-Label-Medikament in die Risikoklasse C, dann muss ebenfalls ein Einzelfallexperiment erfolgen, diesmal vor allem, um die Sicherheit des Patienten zu gewährleisten. Daneben ist es auch wichtig, die Effektgrösse am individuellen Patienten zu dokumentieren.

Unsichere und möglicherweise unwirksame OffLabel-Medikamente sollen nicht eingesetzt werden, auch wenn man sich auf die ärztliche Handlungsfreiheit beruft. Denn ein solches Vorgehen ist mit dem Codex der ärztlichen Berufsethik nicht vereinbar.

\section{Ausblick}

Es ist m. E. ersichtlich, dass die Off-Label-Praxis nicht durch Gesetze oder staatliche Zulassungsbehörden zu kontrollieren ist. Der Patient selbst und wissenschaftliche Institutionen könnten die Kontrolle dieser «wissenschaftlichen Aktivität» übernehmen.

Kompetenzzentren für klinische Forschung wie die 2007 mit der Förderung des Schweizerischen Nationalfonds gegründeten Clinical Trial Units könnten dabei einen wesentlichen Beitrag leisten. Sie haben zwar bislang nur den Auftrag, universitäre Einrichtungen bei der Umsetzung klinischer Forschungsprojekte zu unterstützen, könnten aber in Zukunft Ihre studientechnischen Kenntnisse und ihre Infrastruktur auch der Off-Label-Forschung zur Verfügung stellen.

Sie wären Garant einer umfassenden Qualitätssicherung, die sowohl die Patientensicherheit und die Integrität der erhobenen Daten verbessert [10]. Neben der Studienkoordination könnten sie sogar aus dem Berg von individuellen Daten, die alle durch vergleichbare Experimente gewonnen worden sind, etwas Wertvolles extrahieren (Data mining). Damit könnte sich sogar der Traum erfüllen, verallgemeinerbares Wissen zu erhalten und den echten Nutzen eines Off-Label-Medikaments für adäquate Kosten-Nutzen-Analysen zu bestimmen.

\section{Literatur}

1 Johann Gottlieb Fichte. Ueber den Begriff der Wissenschaftslehre oder der sogenannten Philosophie. Stuttgart: Reclam; 1986.

2 Erickson SH et al. The use of drugs for unlabeled indications. JAMA. 1980:243(15):1543-7.

3 Higgins LC et al. Off-Label Rx. Insurers staring to balk. Med World News. 1988;29(20):22-4.

4 Seiler B, Fries R, Honegger H. Nutzenbewertung für Off-Label-Medikamente. Schweiz Ärztezeitung. 2012;93(19):723-5.

5 Nationalrätin Carobbio Guscetti, Postulat 10.3167 eingereicht am 17.3.2010: Off-Label-Praxis als staatlich kontrollierte Prozedur mit Seitenblick auf Qualitätssicherung und den Leitlinien für eine gute klinische Praxis (ICH-GCP).

6 Rheinberger HJ. Historische Epistemiologie. Hamburg: Junius Verlag; 2007. S.23.

7 Sprecher F, van Spyk B. Regelungsbedarf im Bereich experimenteller Einzelfallbehandlungen an Patienten. www.jusletter.ch 1.2011.

8 Price JD, Evans JG. N-of-1 randomized controlled trials («N-of-1 trials»): singularly useful in geriatric medicine. Age and Ageing 2002:31;227-32

9 Brosteanu $\mathrm{O}$ et al. Risk analysis and risk adapted on-site monitoring in non-commercial clinical trials. Clinical Trials. 2009:6:585-96.

10 Grünig M Weiss C, Meier-Abt P. Die Swiss Clinical Trial Organisation veröffentlicht Qualitätsrichtlinien. Schweiz Ärztezeitung. 2011; 92(44):1690. 